\title{
Full Revelation of Information in Sender-Receiver Games of Persuasion
}

\author{
Jérôme Mathis \\ Toulouse School of Economics (GREMAQ) - University of Toulouse, Manufacture \\ des Tabacs, Aile J.J. Laffont, 21 Allée de Brienne, 31000 Toulouse, France.
}

\begin{abstract}
We provide necessary and sufficient conditions on both players' preferences and information that can be certified for a Sender-Receiver game to possess a separating equilibrium, as well as sufficient conditions for every equilibrium of such a game to be separating. Accordingly, we generalize Seidmann and Winter's [D.J. Seidmann, E. Winter, Strategic information transmission with verifiable messages, Econometrica 65 (1997) 163-170] results to games with partial provability.
\end{abstract}

Key words: Disclosure of certifiable information; Partial provability; Persuasion; Separating equilibrium; Verifiable types

JEL classification: $\mathrm{C} 72, \mathrm{D} 82$

\section{Introduction}

Consider a Sender-Receiver message game: viz. costless, non-binding, faceto-face and one-step unilateral communication game played by an informed interested party (Sender) who sends a message on the basis of his payoffrelevant information to a decision maker (Receiver) who then takes an action. When the set of Sender's available messages vary with his information, we say that some information is certifiable. ${ }^{1}$ What conditions on players' preferences and certifiability are required for the Sender to voluntary reveal his private information to the Receiver ? Said differently, what conditions on players'

Email address: jerome.mathis@TSE-fr.eu (Jérôme Mathis).

1 In this paper, the term "certifiable" is equivalent to the terms "provable" and "verifiable". See subsection 2.2 for a formal definition. 
preferences and message structure are necessary and/or sufficient for such a game to possess a separating equilibrium ?

The literature has addressed this question only for some specific certifiability contexts.

In cheap-talk games the informed party can certify nothing of what he knows (formally, the message space does not depend on the realized type). In such a game, when the Receiver's ideal action (when fully informed) is unique in the Sender's type (e.g., Crawford and Sobel [5]), full revelation occurs if and only if no Sender's type strictly prefers to be misidentified for another. ${ }^{2}$

When the Sender has the ability to certify both all his payoff-relevant information as well as the fact that he is not withholding information (formally, by sending a particular message unavailable to any other type), Milgrom [18], Grossman [12] and Grossman and Hart [13] have shown that under monotonic preferences $^{3}$ all information is revealed in every equilibrium. Existence of such an outcome requires the possibility for the Receiver to use a skeptical (or worst case) inference (i.e., to identify what is the least favorable information for the Sender, and to believe it when he detects or suspects that the Sender withholds piece of information). Seidmann and Winter [21] generalize these existence and uniqueness results by replacing monotonicity with preferences where both players' ideal action strictly increases with the Sender's type, and the bias (i.e., the difference between the two players' ideal actions) either is strictly one-signed in the Sender's type (e.g., Crawford and Sobel [5] ${ }^{4}$ ) or changes in the wrong direction. They also give a single-crossing property on more general preferences that guarantees the existence of a separating equilibrium.

Giovannoni and Seidmann [11] study a Sender-Receiver game where the certifiability is stronger: the Sender has the ability to prove any true event (formally, the Sender can certify all subset of types containing the realized one). They show that such a game possesses a separating equilibrium if and only if no pair of types strictly prefer to be misidentified for another (Giovannoni and

$\overline{2}$ Forges $[7]$ is an example of cheap-talk game satisfying this condition and then possessing a separating equilibrium although players have conflicting preferences. Forges-Koessler ([9], Example 7) is an example of cheap-talk game failing this condition but even so possessing a separating equilibrium (in mixed strategy) because the Receiver's ideal action is not unique in the Sender's type.

3 That is, each Sender's type wants the Receiver to believe that his type is as high (or as low) as possible. More precisely, the Receiver's optimal action increases with the Sender's type, and each Sender's type payoff is monotonic in the Receiver's action.

4 The one-signedness follows from their continuity assumption and their premise of Theorem 1. 
Seidmann's single-crossing property).

While it seems reasonable to consider situations in which an informed agent can certify something, it is often unrealistic to assume that he has the ability to certify everything. The information transmission may be limited by time or technical constraints, the Receiver's ability to verify a proof, or the Sender's ability to prove all true payoff-relevant facts or that he is not withholding others information. Accordingly, we generalize Seidmann and Winter's [21] results to a setting of certifiability that may be partial.

In a context of partial certifiability, Lipman and Seppi [16] provide necessary and sufficient conditions under which full revelation takes place when there are multiple informed agents. They show that the Receiver can extract the information by adopting an inference rule that consists in believing any claim reported by a Sender unless refuted by another. The revelation relies on a competition between Senders with conflicting preferences and cannot apply with only one Sender.

We provide necessary and sufficient conditions on both players' preferences and information that can be certified for the existence of a separating equilibrium. These conditions (Proposition 1) are first that every set of types that can be certified has a worst case inference (Seidmann and Winter's [21] result). And second that every type can separate from all types that strictly prefer to be misidentified as him. For preferences where the bias either is strictly onesigned in the Sender's type (as monotonic preferences) or changes in the wrong direction, we provide conditions on certifiability that ensures existence and uniqueness of the separating equilibrium outcome. The existence condition is that every type can certify that it is at least as high (resp. as low) as it actually is when the Sender's ideal action exceeds (resp. is exceeded by) the Receiver's one. This holds even when contrary to Seidmann and Winter [21], the Sender's ideal action does not increase with the Sender's type (Theorem 1). Uniqueness is guaranteed under the stronger condition that every type can certify a set only containing types for which it prefers to be misidentified (Theorem 2). This last condition also ensures existence of a separating equilibrium under more general preferences satisfying Seidmann and Winter's [21] or Giovannoni and Seidmann's [11] single-crossing property (Theorem 3). Finally, we illustrate the use of our three theorems in an example.

The paper is organized as follows. In Section 2 we present our model describing the general framework and some preliminary definitions. Results, example and applications are offered in Section 3. All proofs have been relegated to an appendix at the end of the paper. 


\section{Model}

A persuasion game, $\Gamma$, is played by a Sender, $S$, who sends a message on the basis of his private information to a Receiver, $R$, who chooses an action after observing the message sent by $S$. Following Seidmann and Winter [21], hereafter referred to as SW, we denote $R$ 's action space by $A$ with generic element $a$. We assume this action set $A$ is a compact interval in $\mathbb{R}$. The Sender's private information or Sender's type, $t$, is realized from a compact interval $\tau \subseteq \mathbb{R}$, with prior distribution $p(t)$. Each player's payoff depends on $R$ 's action and $S$ 's type. We denote player $i$ 's state dependent payoff function, $i=R, S$, by $u^{i}(a ; t)$ where $u^{i}(\cdot ; t)$ is $\mathrm{vN}-\mathrm{M}$ utility function for each type $t \in \tau$.

\subsection{Preferences}

We shall assume throughout that:

A1. Receiver Preferences: For each $t \in \tau, u^{R}(\cdot ; t)$ is concave in $a$, and $a^{R}(t):=\arg \max _{a \in A} u^{R}(a ; t)$ is unique. Furthermore, $a^{R}(\cdot)$ is continuous and increasing in $t$ on $\tau$.

A2. Sender Preferences: For each $t \in \tau, u^{S}(\cdot ; t)$ is strictly concave in a, and $a^{S}(t):=\arg \max _{a \in A} u^{S}(a ; t)$ is unique. Furthermore, $a^{S}(\cdot)$ is continuous in $t$ on $\tau$.

In $\mathrm{A} 1$ and $\mathrm{A} 2$, the action $a^{i}(t) \in A$ denotes the unique maximand of $u^{i}(\cdot ; t)$ on $A$, referring to $i$ 's "ideal action". ${ }^{5}$

\subsection{Messages and certifiability}

The keystone of our analysis is that, contrary to SW we consider a certifiability structure which may be partial. That is, $S$ may be able to certify part of his information to $R$, but unable to certify all of the payoff-relevant information he actually knows. For instance, an expert may present some certified documents (as profits earned, initial endowments, costs, physical proofs) supporting part of his private information to a decision maker, but even by transmitting all

5 A1 is exactly the same $R$ 's preferences assumptions as in SW. In addition to A2, SW's assumptions on $S^{\prime}$ 's preferences requires that $a^{S}(\cdot)$ is (a $C^{1}$ function being) strictly increasing in $t$. 
the documents he has, he cannot prove that he is not withholding additional documents.

An intrinsic certifiability structure requires that the set of available messages depends on $S$ 's private information. We assume the set of available messages may be type-dependent (persuasion game). Let $M(t)$ be the nonempty set of available messages to type $t$. Let $M(\tau):=\cup_{t \in \tau} M(t)$ be the whole set of messages with generic element $m$, and $M$ be the messages structure (that define which information can be certified) defined as the Cartesian product of the set of available messages on the set of types, i.e. $M:=\otimes_{t \in \tau} M(t)$. This structure thus defines the messages intrinsic certifiability and is defined by the communication medium (presentation of documents, certified information, etc...) between both parties. Let $T(m):=\{t \in \tau \mid m \in M(t)\}$ be the set of types $t$ for which the message $m$ is available. Thus, a message $m$ certifies that the realized type is in $T(m)$ and rules out types in $\tau \backslash T(m)$. We shall equivalently say that message $m$ "verifies", "certifies" or "proves" the set $T(m)$. A set $T$ is "verifiable", "certifiable" or "provable" if there is a message $m$ that certifies $T$. We suppose that each certifiable set is closed. Write the game $\Gamma(M)$ as $\left\{M, A, p, u^{R}, u^{S}\right\}{ }^{6}$

\subsection{Solution concept and definitions}

\subsubsection{Solution concept}

We shall analyze the pure strategy perfect Bayesian equilibria of $\Gamma(M)$, which we refer to as its equilibria. Perfect Bayesian equilibrium is particularly well adapted to our situation where a message can intrinsically carry some information on the realized type. In addition to Nash equilibrium it requires that $R$ cannot ignore a proof or evidence transmitted by $S$. More precisely, on receipt of any message $m$ (either on or off the equilibrium path) $R$ 's updated belief must have support in $T(m)$ the set of types for which $m$ is available.

Our main objective is to characterize conditions which ensure the existence of an equilibrium in which all $S$ 's private information is revealed to $R$. We shall say that an equilibrium is fully revealing or separating if there is no identical

$\overline{6 \Gamma(M)}$ can be thought of as many signaling game in which payoffs do not depend directly on messages. Crawford and Sobel [5] is an example of $\Gamma(M)$ where only $\tau$ can be certified (cheap-talk game), $u^{i}(\cdot ; t)$ is strictly concave in $a$, and $a^{i}(\cdot)$ is $C^{1}$ and strictly increasing in $t$ (in their example, $a^{S}(t)=a^{R}(t)+b$ with $b \in \mathbb{R}$ ). Seidmann and Winter [21] is an example of $\Gamma(M)$ where each $t \in \tau$ is certifiable and $a^{S}(\cdot)$ is $C^{1}$ and strictly increasing in $t$. Giovannoni and Seidmann [11] consider a S-R game of persuasion where $\tau$ is finite, every $T \subseteq \tau$ is certifiable, $a^{R}(\cdot)$ is strictly increasing in $t$ and $u^{i}(\cdot ; t)$ is single-peaked in $a$. 
message sent by two different types, so $R$ always learns the true type and chooses $a^{R}(t)$ in response to each message transmitted by $t$, for every $t \in \tau$. We shall denote $T_{\min }$ as $\min (t \in T)$ and $T_{\max }$ as $\max (t \in T)$ for closed $T$.

\subsubsection{Definitions}

Under a perfect Bayesian equilibrium, A1 implies that when receiving a message certifying a set $T, R$ responds by choosing an action $a^{R}(t)$, where $t$ can be chosen as an element of the convex hull of $T$, denoted as $c o(T)$. We shall say such a type $t$ is an inference for $T$.

Existence of a separating equilibrium requires that $R$ plays a strategy giving $S$ the incentive to reveal all his private information. To put such incentive in place, $R$ must respond to each message available to type $t$, by an action getting to $t$ a lower payoff than under action $a^{R}(t)$. Said differently, having observed a message $m, R$ must threaten $S$ with an action $a$ getting to type $t$, for any $t \in T(m)$, a lower payoff than under action $a^{R}(t)$. For this threat to be credible, it requires that action $a$ can be supported by a rational inference. Such an inference is then called a skeptical inference or a worst case inference (wci). Formally, an inference $t^{*} \in c o(T)$ is said to be a worst case inference for $T$ (henceforth $\left.t^{*} \in w c i(T)\right)$ if for all $t \in T$

$$
u^{S}\left(a^{R}\left(t^{*}\right) ; t\right) \leq u^{S}\left(a^{R}(t) ; t\right)
$$

If $t^{*} \in w c i(T)$ and $t^{*} \in T$ then $t^{*}$ is said to be a worst case type for $T(w c t(T))$.

\section{Results}

The remainder is organized as follows. Section 3.1 provides necessary and sufficient conditions for the game to possess separating equilibrium. Section 3.2 uses these preliminary results to establish more tractable sufficient conditions when the game satisfies a given class of preferences that has been widely studied in the literature. For such preferences, Section 3.3 offers sufficient conditions for every equilibrium to be separating. Section 3.4 provides sufficient conditions for separation under a more general class of preferences. Finally, Section 3.5 illustrates the use of all our results by an example.

\subsection{Preliminary results}

Under A1, by definition, if a certifiable set $T$ has no wci then whatever $R$ 's inference $t^{*}$ for $T$, there is a type $t \in T$ who strictly prefers $a^{R}\left(t^{*}\right)$ rather 
than $a^{R}(t)$. The existence of a separating equilibrium under A1 then requires that every certifiable set has a wci. When every type is certifiable, SW has shown that this condition is also sufficient. Indeed, the separating strategies in which each type $t$ sends his self-identifying message $m$ (i.e., such that $T(m)=\{t\}$ ) and $R$ chooses an ideal action supported by a wci for each certified set constitutes an equilibrium.

When dropping SW's assumption that every type is certifiable, even so full revelation may occur at equilibrium. For instance, consider a cheap-talk game of pure common-interest $\left(a^{S}(t)=a^{R}(t)\right.$ for every $\left.t\right)$ with more messages than types. However, the wci condition may not be sufficient to guarantee existence of a separating equilibrium. For instance, in Crawford and Sobel's [5] example only $\tau$ is certifiable and $\tau_{\text {min }}$ is a wct for $\tau$.

The existence of a separating equilibrium does not require that every type $t$ can separate from all other types, but only from types that strictly prefer to be misidentified as type $t$. In other words, it does not require that each type $t$ can send a self-identifying message certifying the set $T=\{t\}$, but only that $t$ can certify a set for which he is a wct. If each type is a wct for the set his separating message certifies then no type has an incentive to deviate to messages on the equilibrium path. To ensure no profitable deviation to messages off the equilibrium path $R$ must threaten by inferring a wci for any certified set.

The following proposition states that under $\mathrm{A} 1, \Gamma(M)$ possess separating equilibrium if and only if, roughly speaking: $(i) R$ can play in a manner that every type $t$ prefers the action $a^{R}(t)$ rather than any action induced by one of his available message; and ( $i i)$ every type $t$ can separate from types that strictly prefer to be misidentified as type $t$.

Proposition 1. If $A 1$, then $\Gamma(M)$ possesses a separating equilibrium if and only if:

(i) Every certifiable set has a wci; and

(ii) Every type can certify (with a different message) a set for which he is a wct.

Condition $(i)$ in Proposition 1 is the SW's necessary and sufficient condition for the existence of a separating equilibrium in games where every type is certifiable. This condition obviously holds if $S$ 's preferences are monotonic in $R$ 's action (e.g., Fishman and Hagerty [8], Grossman [12], Grossman and Hart [13], Koessler [14], Matthews and Postlewaite [17], Milgrom [18], Milgrom and Roberts [19], Shin [22,23]), as for any certifiable set $T$ either $T_{\min }$ or $T_{\max }$ is a wct. 
Condition $(\mathrm{ii})$ in Proposition 1 says that any type $t$ must be able to send a message that certifies a set not containing a type $t^{\prime}$ who would prefer $a^{R}(t)$ rather than $a^{R}\left(t^{\prime}\right)$. Of course, separation requires that such a message can be chosen differently for any type. This condition always holds in persuasion games where every type $t \in \tau$ is certifiable (e.g., Giovannoni and Seidmann [11], Grossman [12], Grossman and Hart [13], Koessler [14], Matthews and Postlewaite [17], Milgrom [18], Milgrom and Roberts [19], Seidmann and Winter [21]) since it is sufficient for type $t$ to send his self-identifying message. Literature has examples of games where this condition fails. This is the case in Fishman and Hagerty $[8]^{7}$ since there is no enough messages, and Cheap-Talk games once there is a type that strictly prefers to be misidentified for another (so this latter type is not a wct for the unique certifiable set $\tau$ ). In a situation where a type possessing more information than another one, can certify all what this latter can certify, condition (ii) requires that the former does not strictly prefer to be misidentified as the latter. The failure of this explains the nonexistence of separating equilibrium in Shin $[22,23] .^{8}$

In the following, using Proposition 1 we shall state three theorems providing sufficient conditions for $\Gamma(M)$ to possess a separating equilibrium (Theorem 1 and Theorem 3) and sufficient conditions for every equilibrium of $\Gamma(M)$ to be separating (Theorem 2). Although the conditions we shall use to imply condition $(i)$ and $(i i)$ could not be treated independently, roughly speaking we shall label condition $(a)$ referring to $(i)$ and condition $(b)$ referring to $(i i)$. That is, condition denoted as $(a)$ will refer to some sufficient conditions on players' preferences and condition denoted as $(b)$ will refer to some sufficient conditions on the message structure which, contrary to SW, only requires partial certifiability. Condition $(a)$ will be the same in Theorem 1 and Theorem 2. Condition $(b)$ will be the same in Theorem 2 and Theorem 3.

7 They assume that $S$ observes $N$ signals, each taking on one of two values, "high" or "low", and that $S$ only can certifiably disclose the realization of one signal. So, there are $2^{N}$ types while there only are $2 N$ available messages.

8 Shin [22] considers two interested parties (a defendant and a plaintiff) whereas Shin [23] consider only one interested party (a firm) and two decision makers (two shareholders). Boiling down to an $S$ - $R$ game, he assumes that $S$ 's preferences are monotonic in $R$ 's action and that $R$ does not know whether $S$ has or not perfectly observed the payoff-relevant state (state of Nature), which is a given value in an interval. He assumes that $S$ observes a randomly chosen interval containing the state of Nature and that he only can prove a larger interval than the one observed, but cannot prove that he has not observed a smaller one. 


\subsection{Existence}

SW's Theorem 1 provides some sufficient conditions on $\Gamma(M)$ to possess a separating equilibrium. Our first theorem generalizes such a result to a partial certifiability setting and more general preferences. Denotes the bias function $B(t)$ as $a^{R}(t)-a^{S}(t)$. A1 and A2 imply that $B(\cdot)$ is continuous in $t$ on $\tau$. $B$ is said to change sign on $T$ if there is a closed interval $I \subseteq T$ such that $B(\cdot)$ is weakly monotonic on $I$ and $B\left(I_{\min }\right) B\left(I_{\max }\right)<0(I$ is said to be a signchanging interval). $B$ is said to change sign $n$ times on $T$ if $n$ is the maximum number of disjoint intervals in $T$ on which $B$ changes sign.

If $S$ 's preferences are monotonic in $R$ 's action or players have Crawford and Sobel's [5] preferences then $B(\cdot)$ does not change sign on $\tau$. Consider for instance that $B(\cdot) \leq 0$ on $\tau$. In this case, A1 and A2 imply that for every certifiable set $T, T_{\min }$ is a wci. In the light of Proposition 1 , a sufficient condition for the existence of a separating equilibrium is that every type $t$ can certify (with a different message) a set for which he is a wct. That is, it suffices that every type $t$ is able to certify a set $T$ for which $T_{\min }=t$. Of course, by substituting $T_{\min }$ for $T_{\max }$ when $B(\cdot) \geq 0$ on $\tau$ we obtain the result. The same reasoning holds if $B(\cdot)$ changes sign once on $\tau$ and $B\left(\tau_{\min }\right)>0 .{ }^{9}$

Theorem 1. If $A 1$ and $A 2$ then $\Gamma(M)$ possesses a separating equilibrium if:

(a) $B(\cdot)$ does not change sign on $\tau$, or $B(\cdot)$ changes sign once on $\tau$ and $B\left(\tau_{\text {min }}\right)>0$; and

(b) Every type $t$ can certify (with a different message) a set $T$ for which $T_{\min }=t$ if there is $t^{\prime}<t$ such that $B\left(t^{\prime}\right)<0$; and $T_{\max }=t$ if there is $t^{\prime}>t$ such that $B\left(t^{\prime}\right)>0$.

Theorem 1 states in particular that in games with monotonic preferences where every type $t \in \tau$ is certifiable (e.g., Grossman [12], Grossman and Hart [13], Koessler [14], Matthews and Postlewaite [17], Milgrom [18], Milgrom and Roberts [19]) a separating equilibrium would still exist under the weaker condition that the Sender rather can certify a subset for which his true type is minimum (resp. maximum) when his ideal action exceeds (resp. is exceeded by) the Receiver's one. The partial certifiability of the games studied

9 Example of preferences satisfying this condition is the following. A patient asks a doctor to diagnose his condition before authorizing him to conduct surgery. Both agents want more intrusive surgery the worse the patient's condition. But the patient wants more surgery than the doctor would like to perform iff he is relatively healthy. 
by Okuno-Fujiwara et al. [20] ${ }^{10}$ and Lanzi and Mathis [15] ${ }^{11}$ satisfy condition (b).

In the following, we shall need to identify the set of actions inciting to deviate from a separating strategy. That is the set of actions getting to type $t$ a higher payoff than under action $a^{R}(t)$. When $a^{R}(t)<a^{S}(t)\left(\right.$ resp. $\left.a^{R}(t)>a^{S}(t)\right)$, write $\tilde{a}(t)$ as $\left\{a \in A \mid a \neq a^{R}(t)\right.$ and $\left.u^{S}(a ; t)=u^{S}\left(a^{R}(t) ; t\right)\right\}$ when $a$ is welldefined, and $\tilde{a}(t)=+\infty$ (resp. $\tilde{a}(t)=-\infty)$ otherwise. When $a^{R}(t)=a^{S}(t)$, write $\tilde{a}(t)$ as $a^{R}(t)$. A2 implies that $\tilde{a}(t)$ is unique. Notice that if $u^{S}(\cdot ; t)$ were symmetric round $a^{S}(t)$ then $\tilde{a}(t)=2 a^{S}(t)-a^{R}(t)$.

\subsection{Uniqueness}

From now, we shall generalize SW's Theorem 3 which provides some sufficient conditions for every equilibrium to be separating. In addition to the premises of Theorem 1, assume that $a^{R}(\cdot)$ is strictly increasing in $t$ on $\tau$. So for any certifiable set $T$ which is nonsingleton, whenever $B(\cdot)>0$ on $T$, type $T_{\min }$ strictly prefers action $a^{R}\left(T_{\min }\right)$ rather than $a^{R}(t)$ for all $t$ in $c o(T) \backslash\left\{T_{\min }\right\}$, and so rather than any $R$ 's equilibrium response to a message certifying a set $T$. On the other hand, any $R$ 's equilibrium response to a message $m^{\prime}$ that certifies a set $T^{\prime}$ with $T_{\max }^{\prime}=T_{\min }$ and $a^{R}\left(T_{\min }^{\prime}\right)>\tilde{a}\left(T_{\min }\right)$, is an action $a\left(m^{\prime}\right)$ that gets a higher (or equal) payoff to type $T_{\min }$, than does action $a^{R}\left(T_{\min }\right)$. So if type $T_{\min }$ can certify set $T^{\prime}$, doing so always constitutes a profitable unilateral deviation from certifying set $T$. A similar reasoning applies with type $T_{\max }$ when $B(\cdot)<0$ on $T$. Thus, $S$ 's equilibrium strategy contains a pooling set of types sending the same message. Any equilibrium is then separating. As for Theorem 1, we shall show that the same reasoning holds if $B(\cdot)$ changes sign once on $\tau$ and $B\left(\tau_{\min }\right)>0$.

Theorem 2. Suppose $a^{R}(\cdot)$ is strictly increasing in $t$ on $\tau$. If A1, A2 and:

\footnotetext{
${ }^{10}$ They consider a game with multiple agents. They assume that each agent can send a message consisting of a set of truthful lower bounds for the signal he has observed. They show that if agents' payoffs are positive-monotone in beliefs then every equilibrium is separating.

11 They consider a situation where $R$ relies on the report of $S$ prior to decide whether to undertake or not a certain project. The higher is the value of the observed state the more the state is conveying favorable information sustaining the project. Information contained in the report is partially verifiable in the sense that $S$ can suppress favorable information but he cannot exaggerate it. They assume that conflicting preferences only occur for medium state values. The ideal players' action for such values then identifies the more eager agent for the project to be undertaken. In particular, they show that there is a separating equilibrium if and only if $S$ is the more eager agent.
} 
(a) $B(\cdot)$ does not change sign on $\tau$, or $B(\cdot)$ changes sign once on $\tau$ and $B\left(\tau_{\min }\right)>0$; and

(b') Every type $t$ can certify (with a different message) a set $T$ for which $T_{\min }=t$ and $a^{R}\left(T_{\max }\right)<\tilde{a}(t)$ if $B(t)<0 ; T=\{t\}$ if $B(t)=0$; and $T_{\max }=t$ and $a^{R}\left(T_{\min }\right)>\tilde{a}(t)$ else,

then every equilibrium of $\Gamma(M)$ is separating.

Theorem 2 states in particular that in games where the Sender wants to maximize the magnitude of the Receiver's action (e.g., Fishman and Hagerty [8], Grossman [12], Grossman and Hart [13], Koessler [14], Matthews and Postlewaite [17], Milgrom [18], Milgrom and Roberts [19], Okuno-Fujiwara et al. [20], Shin [23]), every equilibrium is separating whenever the Sender can certify a subset for which his true type is minimum (as with such preferences $B(t)<0$ then implies $\tilde{a}(t)=+\infty)$.

\subsection{Existence under more general preferences}

Now, under A1 and A2 we shall provide sufficient conditions on $\Gamma(M)$ that guarantee the existence of a separating equilibrium even when players' preferences may fail the premises of Theorem 1 and 2, but satisfy a single-crossing property. The single-crossing property we use for a set $T$ excludes the possibility that $T$ contains any pair of types which strictly prefer to be misidentified for another. ${ }^{12},{ }^{13}$

Single-crossing property for set $T$. If $u^{S}\left(a^{R}\left(t_{1}\right) ; t_{1}\right)<u^{S}\left(a^{R}\left(t_{2}\right) ; t_{1}\right)$ for any pair $t_{1}, t_{2} \in T$, then $u^{S}\left(a^{R}\left(t_{1}\right) ; t_{2}\right) \leq u^{S}\left(a^{R}\left(t_{2}\right) ; t_{2}\right)$.

Our next result will exploit the fact that if a type $t$ can certify a set $T$ that both satisfies the single-crossing property (Theorem 3 condition $\left(a_{1}\right)$ ) and only contains types either for which $t$ strictly prefers to be misidentified or for which $R$ 's ideal action is the same (Theorem 3 condition $\left(b^{\prime}\right)$ ), then $t$ is a wct for $T$.

$\overline{12}$ This property for set $\tau$ is equivalent to the one in Giovannoni-Seidmann [11] if $a^{R}(\cdot)$ is strictly increasing in $t$. The authors show that this is a necessary and sufficient condition for games where every set of types is verifiable to possess a separating equilibrium. Here, when dropping such an assumption on the information that can be certified, the single-crossing property is obviously no longer necessary (consider for instance, a game in which one pair of types violates this property but is unable to certify a common set not containing a wct).

${ }^{13}$ It can be showed that this property is implied by SW's single-crossing property if $a^{S}(\cdot)$ is strictly increasing in $t$. 
Rather than assuming the single-property for any pair of types, we only need to assume it for pair of types who have a common available message (Theorem 3 condition $\left.\left(a_{1}\right)\right)$. (This distinction plays a role only if $\tau$ is not certifiable.) To establish the proof, we shall use an inductive argument on the number of sign changing of $B(\cdot)$ requiring that $B(\cdot)$ changes sign finitely often on $\tau$ (Theorem 3 condition $\left.\left(a_{2}\right)\right)$.

Theorem 3. If $A 1$ and $A 2$ then $\Gamma(M)$ possesses a separating equilibrium if: $\left(a_{1}\right)$ Every certifiable set satisfies the single-crossing property; and $\left(a_{2}\right) B(\cdot)$ changes sign finitely often on $\tau$; and

(b') Every type $t$ can certify (with a different message) a set $T$ for which $T_{\min }=t$ and $a^{R}\left(T_{\max }\right)<\tilde{a}(t)$ if $B(t)<0 ; T=\{t\}$ if $B(t)=0$; and $T_{\max }=t$ and $a^{R}\left(T_{\min }\right)>\tilde{a}(t)$ else.

Without condition $\left(a_{2}\right)$ requiring that $B(\cdot)$ changes sign finitely often on $\tau$, our Theorem 3 would be the generalization of the SW's Theorem $2 .{ }^{14}$

\subsection{Example}

$\tau=A=[0,1], \Gamma(M)$ such that preferences represented by:

$$
\begin{gathered}
u^{R}(a ; t)=-[a-t]^{2}, \\
u^{S}(a ; t)=-[a-(\alpha+\beta t)]^{2}, \beta \neq 1,
\end{gathered}
$$

As $u^{S}(\cdot ; t)$ is symmetric round $a^{S}(t)$, on $A$ we have $\tilde{a}(t):=2 a^{S}(t)-a^{R}(t)$; so

$$
\tilde{a}(t)=\left\{\begin{array}{c}
-\infty \text { if } \alpha+\beta t<\frac{t}{2} \\
+\infty \text { if } \alpha+\beta t>\frac{t+1}{2} \\
2 \alpha+(2 \beta-1) t \text { else }
\end{array}\right.
$$

$B(\cdot)$ has a unique zero at $t^{*}=\frac{\alpha}{(1-\beta)}$ (if $t^{*} \notin(0,1)$ then $B(\cdot)$ does not change $\operatorname{sign}$ on $\tau$ ). If $\alpha \leq 0$ or $\alpha+\beta \geq 1$ then, from Theorem 1 , there is a separating equilibrium if every $t$ can certify (with a different message) a set $T$ satisfying: $T_{\max }=t$ when $(1-\beta) t>\alpha$; and $T_{\min }=t$ when $(1-\beta) t<\alpha$. In addition, from Theorem 2, every equilibrium is separating if $t$ can certify (with a different message) a set $T$ satisfying: $T_{\max }=t$ and $T_{\min } \geq \tilde{a}(t)$ when $(1-\beta) t>\alpha$; $T=\{t\}$ when $t=t^{*}$; and $T_{\min }=t$ and $T_{\max } \leq \tilde{a}(t)$ else. From Theorem

\footnotetext{
${ }^{14}$ We can obtain the generalization of SW's Theorem 2 by replacing our singlecrossing property by theirs and restricting our $S$ 's preferences to theirs (that is, requiring in addition to $\mathrm{A} 2$ that $a^{S}(\cdot)$ is $C^{1}$ and strictly increasing in $t$ ).
} 
3 , this latter condition ensures that there is a separating equilibrium when $\alpha>0, \alpha+\beta<1$ and $\beta \geq-1(\beta<-1$ would violate the single-crossing property for set $\tau$ ).

\subsection{Applications}

When an informed agent does not have the ability to certify all his private information we have shown that full revelation requires an additional condition: every type can separate from types that prefer to pool with him. This condition is strong. But the decision-maker (Receiver) may ask the informed party (Sender) to provide appropriate available documents so that this condition holds. Here are some applications.

A seller can ask a budget-constrained buyer to post a bond equal to his reported budget in order to prevent him from overstating it (see, e.g., Che and Gale [4]). Traders in an exchange economy can be required to deposit collateral for each order preventing them from over-reporting their initial endowments (see, e.g., Forges et al. [10]). Financial reporting companies can be compelled by legislation (see, e.g., Sarbanes-Oxley Act of 2002) to annually issue a statement identifying the framework used by management to evaluate the effectiveness of the company's internal control in order to prevent them from misleading investors. A court can ask a creditor declaring that a debtor paid him with a bounced check to present a bank notice stating that the debtor's check was returned due to insufficient funds, otherwise giving weight to negative evidence (see, e.g., Bull and Watson [2]). Consumers may turn toward eco-labeled products to prevent both industrial and agricultural sectors from withholding the environmental externalities associated with their production process (see, e.g., Cason and Gangadharan [3]). Similarly, investors may turn toward issues that are underwritten by reputable investment banks to detect firms that are significantly less risky (see, e.g., Fang [6]). A firm hoping to appropriate an innovation may be compelled by the patent granting authority to present the new technical knowledge it possess (see, e.g., d'Aspremont et al. [1]).

\section{Acknowledgements}

This paper is the fourth chapter of my Ph.D. thesis. I thank Françoise Forges, Frédéric Koessler and an anonymous referee for very helpful suggestions. I also thank seminar participants at U. Paris 1, U. Cergy-Pontoise, Paris Game 
Theory Seminar (Institut Henri Poincaré), the GREQAM Summer School (Aix-en-Provence) for useful comments. Financial support from an ACI grant by the French Ministry of Research is gratefully acknowledged.

\section{Appendix}

Proof of Proposition 1. Formally, condition (ii) write as: there is an injection $i$ from $\tau$ to $M(\tau)$ such that $i(t) \in M(t)$ and $t \in \operatorname{wct}(T(i(t)))$.

Sufficiency. Suppose $(i)$ and (ii) hold. The following strategies constitute a separating equilibrium. Each type $t$ sends $i(t)$ (as considered in the formalization of $(i i)$ ), to which $R$ responds with $a^{R}(t)$ (injection $i$ ensures that separation holds). In addition, $R$ responds to any message $m$ with $a^{R}\left(t^{*}\right)$, where $t^{*}$ is a wci for $T(m)((i) \Longrightarrow w c i(T(m)) \neq \varnothing)$; so no type would have an incentive to deviate.

Necessity. Suppose, contrary to the proposition, that there is a separating equilibrium and either $(i)$ or $(i i)$ does not hold. First, suppose that $(i)$ does not hold: there is a message $m \in M(\tau)$ with $w \operatorname{ci}(T(m))=\varnothing$. Thus for each $t^{*} \in \operatorname{co}(T(m))$, there is $t \in T(m)$ such that $u^{S}\left(a^{R}\left(t^{*}\right) ; t\right)>u^{S}\left(a^{R}(t) ; t\right)$. Such a type $t$ would has an incentive to deviate to $m \in M(t)$ as, by supposition his equilibrium message induces $a^{R}(t)$, a contradiction. Second, suppose that (ii) does not hold. If there is a type $t \in \tau$ who sends a message $m \in M(t)$, certifying the set $T(m)$ for which $t$ is not a wct then, by definition, there is a type $t^{\prime} \in T(m)$ such that $u^{S}\left(a^{R}(t) ; t^{\prime}\right)>u^{S}\left(a^{R}\left(t^{\prime}\right) ; t^{\prime}\right)$. Such a type $t^{\prime}$ would then has an incentive to send $m \in M\left(t^{\prime}\right)$, as by supposition his equilibrium message induces $a^{R}\left(t^{\prime}\right)$ while message $m$ induces $a^{R}(t)$. If each type $t$ sends $i(t)$ (as considered in the formalization of $(i i)$ ), but $i$ is not an injection from $\tau$ to $M(\tau)$ then there are two different types $t_{1}, t_{2} \in \tau$, with $i\left(t_{1}\right)=i\left(t_{2}\right)$. So, $R$ cannot distinguish between $t_{1}$ and $t_{2}$.

Determining whether a set has a wci may not be straightforward. The use of the following lemma in the proof of Theorems 1 and 3 will allow us to simplify the problem. A2 implies that the set of actions that a type $t$ strictly prefers to $a^{R}(t)$ write as the intersection between $A$ and the open interval with endpoints $a^{R}(t)$ and $\tilde{a}(t)$. By definition of a wci $t^{*}$ for $T, a^{R}\left(t^{*}\right)$ does not belong to this set of actions. Lemma 1 simply says that action $a^{R}\left(t^{*}\right)$ is then out of the open interval with endpoints $a^{R}(t)$ and $\tilde{a}(t)$.

Lemma 1. If A2, then an inference $t^{*}$ for $T$ is a wci for $T$ if and only if for each $t \in T$ we have

$$
\left[a^{R}\left(t^{*}\right) \leq \min \left\{a^{R}(t), \tilde{a}(t)\right\} \text { or } a^{R}\left(t^{*}\right) \geq \max \left\{a^{R}(t), \tilde{a}(t)\right\}\right]
$$


Proof of Lemma 1. Necessity. Let $t^{*}$ be a wci for $T$. By definition, $a^{R}\left(t^{*}\right) \in A$ and for every $t \in T$, we have $u^{S}\left(a^{R}\left(t^{*}\right) ; t\right) \leq u^{S}\left(a^{R}(t) ; t\right)$. Let $t \in T$. Let us distinguish between three cases:

Case 1: $a^{R}(t)<a^{S}(t)$. By A2 and by definition of $\tilde{a}(t)$, we firstly have $a^{R}(t)<$ $a^{S}(t)<\tilde{a}(t)$, and secondly either $a^{R}\left(t^{*}\right) \leq a^{R}(t)=\min \left\{a^{R}(t), \tilde{a}(t)\right\}$ or $[\tilde{a}(t)<$ $+\infty$ and $\left.a^{R}\left(t^{*}\right) \geq \tilde{a}(t)=\max \left\{a^{R}(t), \tilde{a}(t)\right\}\right]$.

Case 2: $a^{R}(t)>a^{S}(t)$. An analogous argument to Case 1 allows us to conclude. Case 3: $a^{R}(t)=a^{S}(t)$. By definition of $\tilde{a}(t)$, we have $\min \left\{a^{R}(t), \tilde{a}(t)\right\}=$ $\max \left\{a^{R}(t), \tilde{a}(t)\right\}$, which allows us to conclude.

Sufficiency. Let $t \in T$ and $t^{*} \in \operatorname{co}(T)$. Suppose $a^{R}\left(t^{*}\right) \leq \min \left\{a^{R}(t), \tilde{a}(t)\right\}$. From $a^{R}\left(t^{*}\right) \in A$ which is a bounded set we obtain $\tilde{a}(t)>-\infty$. By definition of $\tilde{a}(t)$ and from A2, for every $a \in A$, if $a \leq \min \left\{a^{R}(t), \tilde{a}(t)\right\}$ then $u^{S}(a ; t) \leq$ $u^{S}\left(a^{R}(t) ; t\right)$. In particular whenever $a=a^{R}\left(t^{*}\right)$. An analogous argument allows us to conclude when $a^{R}\left(t^{*}\right) \geq \max \left\{a^{R}(t), \tilde{a}(t)\right\}$.

Proof of Theorem 1. In order to establish existence of a separating equilibrium under A1, it suffices to prove that Proposition 1 conditions $(i)$ and $(i i)$ hold. A2 will allow us to use Lemma 1. Let us first show that Proposition 1 condition $(i)$ holds.

Suppose $(a)$. Fix a certifiable set $T \subseteq \tau$. If $B(\cdot)$ does not change sign on $T$ then we either have $\tilde{a}(t) \geq a^{S}(t) \geq a^{R}(t) \forall t \in T$, or $\tilde{a}(t) \leq a^{S}(t) \leq a^{R}(t)$ $\forall t \in T$. That is, by Lemma 1 , either $T_{\min }$ or $T_{\max }$ is a wct as under A1 $a^{R}(\cdot)$ is increasing in $t$ on $\tau$. If $B(\cdot)$ changes sign on $T$ then there is an inference $t^{*} \in \operatorname{co}(T)$ such that $a^{S}\left(t^{*}\right)=a^{R}\left(t^{*}\right)$, as A1 and A2 imply that $B(\cdot)$ is continuous in $t$ on $\tau$. From $(a)$, such a $t^{*}$ can be chosen such that there is no pair $\left(t^{\prime}, t^{\prime \prime}\right)$ with $t^{\prime}<t^{*}<t^{\prime \prime}$ satisfying $B\left(t^{\prime}\right)<0<B\left(t^{\prime \prime}\right)$. Using Lemma 1 , it is then easily checked that such a $t^{*}$ is a wci for $\tau$ and hence for $T$. Therefore, Proposition 1 condition $(i)$ holds.

Now, let us show that condition $(i i)$ holds. Fix a type $t \in \tau$. Let us distinguish between three cases:

Case 1: There is a type $t^{\prime}>t$ such that $B\left(t^{\prime}\right)>0$. From $(b)$, type $t$ can certify a set $T$ for which $T_{\max }=t$. By $(a)$, for every $t^{\prime \prime}<t, B\left(t^{\prime \prime}\right) \geq 0$. So $\tilde{a}\left(t^{\prime \prime}\right) \leq a^{R}\left(t^{\prime \prime}\right) \leq a^{R}\left(T_{\max }\right)=a^{R}(t)$ as under A1 $a^{R}(\cdot)$ is increasing in $t$ on $\tau$. By Lemma $1, t$ is then a wct for $T$.

Case 2: There is a type $t^{\prime}<t$ such that $B\left(t^{\prime}\right)<0$. By substituting $T_{\max }$ for $T_{\min }$, a similar argument to Case 1 applies.

Case 3: There is neither $t^{\prime}>t$ such that $B\left(t^{\prime}\right)>0$ nor $t^{\prime}<t$ such that $B\left(t^{\prime}\right)<0$. Thus, for every $t^{\prime}>t$ we have $a^{R}\left(t^{\prime}\right) \leq a^{S}\left(t^{\prime}\right)$. By definition of $\tilde{a}\left(t^{\prime}\right)$, A2 implies that $a^{R}\left(t^{\prime}\right) \leq a^{S}\left(t^{\prime}\right) \leq \tilde{a}\left(t^{\prime}\right)$. So $a^{R}(t) \leq \min \left\{a^{R}\left(t^{\prime}\right), \tilde{a}\left(t^{\prime}\right)\right\}$. Also, for any type $t^{\prime \prime}<t$, we obtain $a^{R}(t) \geq \max \left\{a^{R}\left(t^{\prime}\right), \tilde{a}\left(t^{\prime}\right)\right\}$. Under A2, Lemma 1 allows us to assert that $t$ is a wct for $\tau$ and then for any set he can certify. Consequently, every type can certify a set for which he is a wct. From $(b)$, the possibly use of a different message to certify those sets implies Proposition 1 condition ( $i i)$ holds. 
Proof of Theorem 2. Suppose contrary to the theorem, that $T$ is a pooling set in some equilibrium. As we are studying pure equilibria, define $a^{R}(T)$ as $\arg \max _{a \in A} \int_{T} u^{R}(a ; t) d p(t)$; under A1, $a^{R}(T)$ is well-defined. If $a^{R}(\cdot)$ is strictly increasing in $t$ then $a^{R}\left(T_{\min }\right)<a^{R}(T)<a^{R}\left(T_{\max }\right)$. Condition $(a)$ implies that $B\left(T_{\min }\right) \geq 0$ or $B\left(T_{\max }\right) \leq 0$. So, whatever $R$ 's equilibrium strategy, under A2 type $T_{\min }$ or $T_{\max }$ would strictly prefer to deviate by certifying a set as defined in $\left(b^{\prime}\right)$ over certifying $T$, a contradiction.

In the proof of Theorem 3, we shall use the following definitions. If $B(\cdot)$ changes sign finitely often, we shall say that $t_{-}$(resp. $\left.t_{+}\right)$is the first (resp. last) sign change on $T$ if it is the minimal (resp. maximal) zero $t$ of $B(\cdot)$ on $T$ which is in a sign-changing interval in $T$, and such that $\left[T_{\min }, t\right]\left(\operatorname{resp} .\left[t, T_{\max }\right]\right)$ does not contain a sign-changing interval.

Proof of Theorem 3. As in the proof of Theorem 1, under A1 and A2 it suffices to prove that the premises of Proposition 1 hold, using Lemma 1 . We shall start by proving the following lemma to show that conditions $\left(a_{1}\right)$ and $\left(a_{2}\right)$ imply Proposition 1 condition $(i)$.

Lemma 2. If $A 1$ and $A 2$ then any certifiable set satisfying the single-crossing property and on which $B(\cdot)$ changes sign finitely often has a wci.

Proof of Lemma 2. We shall use the following inductive argument.

Inductive Hypothesis. If every compact interval satisfying the single-crossing property on which $B(\cdot)$ changes sign $n$ times has a wci, then every compact interval satisfying the single-crossing property on which $B(\cdot)$ changes sign $n+1$ times has a wci.

Proof. Let $T \subseteq \tau$ be a compact interval which satisfies the single-crossing property. In the first part of the proof of Theorem 1 we have shown that if $B(\cdot)$ does not change sign on $T$ then $T$ has a wci. Let $B(\cdot)$ change sign $n+1$ times on $T$. Let us set $t_{-}\left(t_{+}\right)$as the first (last) sign change on $T$. By definition, $B(\cdot)$ changes sign $n$ times on $\left[t_{-} ; T_{\max }\right]$ and on $\left[T_{\min } ; t_{+}\right]$. The Inductive Hypothesis implies that each interval $\left[t_{-} ; T_{\max }\right]$ and $\left[T_{\min } ; t_{+}\right]$has a wci. We shall distinguish between three cases.

Case 1: $B\left(T_{\min }\right) \geq 0$. Let $t^{*} \in w c i\left(\left[t_{-} ; T_{\max }\right]\right)$. By definition of $t_{-}$, for all $t \in\left[T_{\min } ; t_{-}\right]$we have $B(t) \geq 0$. So A1 and A2 imply that $u^{S}\left(a^{R}\left(t^{*}\right), t\right) \leq$ $u^{S}\left(a^{R}(t), t\right)$. Hence $t^{*} \in w c i(T)$.

Case 2: $B\left(T_{\max }\right) \leq 0$. Analogously any wci for the interval $\left[T_{\min } ; t_{+}\right]$is a wci for $T$.

Case 3: $B\left(T_{\min }\right)<0<B\left(T_{\max }\right)$. We proceed by contradiction. Suppose that $T$ has no wci. Let $t^{*} \in w c i\left(\left[T_{\min } ; t_{+}\right]\right)$. By Lemma 1 , there is a type 
$t_{1} \in\left[t_{+} ; T_{\max }\right]$ such that $\tilde{a}\left(t_{1}\right)<a^{R}\left(t^{*}\right)<a^{R}\left(t_{1}\right)$. As $B(\cdot) \geq 0$ on $\left[t_{+} ; T_{\max }\right]$ and $T_{\max }$ is not a wci for $T$, by Lemma 1 , there is a type $t_{2} \in\left[T_{\min } ; t_{+}\right]$such that $a^{R}\left(t_{2}\right)<a^{R}\left(T_{\max }\right)<\tilde{a}\left(t_{2}\right)$. As $t^{*} \in w c i\left(\left[T_{\min } ; t_{+}\right]\right)$, we have $a^{R}\left(t^{*}\right) \leq a^{R}\left(t_{2}\right)$. Thus, $\tilde{a}\left(t_{1}\right)<a^{R}\left(t^{*}\right) \leq a^{R}\left(t_{2}\right) \leq a^{R}\left(t_{1}\right) \leq a^{R}\left(T_{\max }\right)<\tilde{a}\left(t_{2}\right)$. We again shall distinguish between two cases.

Case 3.1: $a^{R}\left(t_{2}\right)<a^{R}\left(t_{1}\right)$. Hence, from the previous inequality $t_{1}$ and $t_{2}$ violates the single-crossing property, a contradiction.

Case 3.2: $a^{R}\left(t_{2}\right)=a^{R}\left(t_{1}\right)$. As $t_{1}$ is not a wci for $T$, by Lemma 1 , there either is $t_{3} \in\left[t_{1} ; T_{\max }\right]$ such that $\tilde{a}\left(t_{3}\right)<a^{R}\left(t_{1}\right)<a^{R}\left(t_{3}\right)$ or $t_{4} \in\left[T_{\min } ; t_{1}\right]$ such that $a^{R}\left(t_{4}\right)<a^{R}\left(t_{1}\right)<\tilde{a}\left(t_{4}\right)$. So, we either have $\tilde{a}\left(t_{3}\right)<a^{R}\left(t_{2}\right)=a^{R}\left(t_{1}\right)<a^{R}\left(t_{3}\right) \leq$ $a^{R}\left(T_{\max }\right)<\tilde{a}\left(t_{2}\right)$ or $\tilde{a}\left(t_{1}\right)<a^{R}\left(t^{*}\right) \leq a^{R}\left(t_{4}\right)<a^{R}\left(t_{1}\right)<\tilde{a}\left(t_{4}\right)$. Hence, either $\left(t_{2}\right.$ and $\left.t_{3}\right)$ or $\left(t_{1}\right.$ and $\left.t_{4}\right)$ violates the single-crossing property, a contradiction.

Theorem 1 and the Inductive Hypothesis then imply Lemma 2.

To conclude, notice that conditions $\left(a_{1}\right)$ excludes the possibility that any certifiable set contains a pair of types strictly preferring to be misidentified for another. While condition $\left(b^{\prime}\right)$ states that every type can certify, by sending a different message, a set which only contains types inducing the same $R$ 's ideal action or for which he strictly prefers to be misidentified. Therefore, conditions $\left(a_{1}\right)$ and $\left(b^{\prime}\right)$ imply Proposition 1 condition $(i i)$.

\section{References}

[1] d'ASPREMONT, C., BHATTACHARYA, S., GERARD-VARET, L.-A., (2000): "Bargaining and sharing innovative knowledge," Review of Economic Studies 67, pp. 255-271.

[2] BULL, J., WATSON, J., 2004. Evidence disclosure and verifiability. J. Econ. Theory 118, 1-31.

[3] CASON T. N. and GANGADHARAN L., "Environmental Labeling and Incomplete Consumer Information in Laboratory Markets", Journal of Environmental Economics and Management 43 (2002), pp. 113-134.

[4] Y. CHE, I. GALE, The optimal mechanism for selling to a budget-constrained buyer, J. Econ. Theory 92 (2000)

[5] CRAWFORD, V. and J. SOBEL (1982): "Strategic Information Transmission," Econometrica, 50, pp. 1431-1451.

[6] FANG, L.H., "Investment Bank Reputation and the Price and Quality of Underwriting Services", Journal of Finance, Vol. 60, No. 6, pp. 2729-2761, December 2005 
[7] FORGES, F. (1990): "Equilibria with communication in a job market example," Quarterly Journal of Economics 105, 375-398.

[8] FISHMAN, M., and K. HAGERTY (1990): "The Optimal Amount of Discretion to Allow in Disclosure," Quarterly Journal of Econ., 427-444.

[9] FORGES, F. and F. KOESSLER (2007): "Transmission stratégique de l'information et certification," Annales d'Économie et de Statistiques, forthcoming.

[10] FORGES, F., MERTENS, J.-F., VOHRA, R., (2002): "The ex ante incentive compatible core in the absence of wealth effects," Econometrica 70 (5), pp. 1865-1892.

[11] GIOVANNONI, F. and D.J. SEIDMANN (2007): "Secrecy, Two-Sided Bias and the Value of Evidence," Games and Economic Behavior, Vol. 59, pp. 296-315.

[12] GROSSMAN, S.J. (1981): "The informational role of warranties and private disclosure about product quality," J. Law Econom. 24, 461-483.

[13] GROSSMAN, S.J. and O.D. HART (1980): "Disclosure laws and takeover bids," J. Finance. 35, 323-334.

[14] KOESSLER, F. (2003): "Persuasion games with higher-order uncertainty," J. Econ. Theory 110, 393-399.

[15] LANZI, T. and J. MATHIS (2007): "Consulting an Expert with Potentially Conflicting Preferences," Theory and Decision, forthcoming.

[16] LIPMAN, B.L. and D.J. SEPPI, (1995): "Robust Inference in Communication Games with Partial Provability," J. Econ. Theory 66, pp. 370-405.

[17] MATTHEWS, S. and A. POSTLEWAITE (1985): "Quality testing and disclosure," Rand Journal of Economics 16, 328-340.

[18] MILGROM, P. (1981): "Good news and bad news: representation theorems and applications," Bell J. Econom. 12 380-391.

[19] MILGROM, P. and J. ROBERTS (1986): "Relying on the Information of Interested Parties," Rand Journal of Economics 17, 18-32.

[20] OKUNO-FUJIWARA, M., A. POSTLEWAITE, and K. SUZUMARA (1990): "Strategic Information Revelation," Rev. Econ. Stud. 57, 25-47.

[21] SEIDMANN, D.J. and E. WINTER (1997): "Strategic Information Transmission with Verifiable Messages," Econometrica 65, 163-170.

[22] SHIN, H.S. (1994a): "The Burden of Proof in a Game of Persuasion," J. Econ. Theory 64, 253-264.

[23] SHIN, H.S. (1994b): "News Managements and the Value of Firms," Rand Journal of Economics 25, 58-71. 